\begin{tabular}{|c|l|}
\hline III Simpósio Paranaense de Modelagem, & Artigo: 19 \\
$\begin{array}{c}\text { Simulaçăo Controle de Processos } \\
\text { ISSN : 1984-7521 }\end{array}$ & Páginas: 138-144 \\
\cline { 2 - 2 } &
\end{tabular}

\title{
REVISÃO SOBRE CONTROLADORES PID DE ORDEM FRACIONÁRIA
}

\author{
Gabriel Filetti Martins, Guilherme Koiti Tanaka Sassaki, Cid Marcos G. Andrade* \\ 1- Universidade Estadual de Maringá - UEM - PR, cmgandrade@ uem.br

\section{Resumo} \\ Os métodos de controle estão cada vez mais presentes no campo industrial, tal fato gera uma \\ constante necessidade de inovação sobre o tema. Este trabalho apresenta uma revisão sobre controladores \\ PID de ordem fracionária. Inicialmente é apresentada a história do cálculo fracionário e suas definições \\ mais utilizadas em sistemas de controle. Posteriormente é realizada uma análise sobre diversos trabalhos \\ envolvendo controladores PID de ordem fracionária (FOPID), buscando mostrar o desempenho superior \\ deste tipo de controlador em relação ao PID convencional. Contudo, ainda são necessárias pesquisas \\ sobre o tema a fim de precisar com mais detalhes as características de tais controladores.
}

Palavras-chave: Controlador PID de ordem fracionária, Cálculo fracionário, Sistemas de controle, Controlador PID convencional.

\section{Introdução}

O cálculo fracionário representa hoje uma nova alternativa, ainda mais precisa, para descrever objetos e fenômenos. Apesar de ser um tema relativamente novo no campo industrial e da pesquisa, o cálculo fracionário é uma ferramenta matemática antiga, tendo nascido concomitantemente ao cálculo convencional [1].

Suas aplicações vêm despertando a atenção de pesquisadores ao redor do mundo, causando um crescimento acelerado no seu desenvolvimento e, consequentemente, de sua utilização em processos industriais.

No campo do controle, o cálculo fracionário foi introduzido inicialmente por $\mathrm{M}$. Axtell em 1990 [2], desde então novos métodos e técnicas de sintonia vem sendo desenvolvidas. Neste artigo de revisão será apresentada uma breve história sobre cálculo fracionário, bem como suas definições matemáticas e sua utilização em sistemas de controle. Paralelamente a isto, será feita uma breve introdução sobre controladores PID de ordem fracionária, discriminando suas definições, equações e métodos de sintonia.

\section{Cálculo Fracionário}

\subsection{História do Cálculo Fracionário}

Apesar de ter aproximadamente 300 anos de história, o cálculo fracionário é um tema relativamente novo no campo da pesquisa. Atribui-se a primeira ideia a respeito do tema a uma carta escrita por L'Hôpital perguntando a G. Leibniz qual seria o significado de uma derivada de ordem $\mathrm{n}=0,5$. Em resposta, Leibniz previu que, consequências úteis a respeito deste tema seriam retiradas algum dia. Por esta razão, Leibniz é considerado o pai do cálculo fracionário [1].

Inicialmente o cálculo fracionário se restringia a estudos matemáticos teóricos, desenvolvidos por brilhantes matemáticos da época, como Euler, Laplace, Lagrange, Fourier, entre outros, porém nenhuma aplicação foi desenvolvida no primeiro século de existência do tema. Somente em 1823, N.Abel desenvolveu a primeira aplicação do cálculo fracionário ao descobrir que a solução do problema de tautocronia poderia ser obtida utilizando-se integrais de ordem 0,5 [1].

O primeiro estudo sistemático sobre cálculo fracionário foi desenvolvido pro Joseph Liouville em 1832 que foi atraído ao tema após comentários feitos por Laplace e Fourier e pelo trabalho de N.Abel [3]. Já a primeira utilização de cálculo fracionário em sistemas de controle foi feita por M. Axtell em 1990, onde ele introduziu operadores utilizando a transformada de Laplace. Apesar de ser um trabalho um tanto superficial na análise da utilização em sistemas de controle, Axtell, recomendou a realização de 
pesquisas a fim de identificar os impactos que sistemas de ordem fracionária teriam no campo do controle, mostrando assim o potencial de utilização do cálculo fracionário em sistemas de controle [2].

\subsection{Definições}

Existem inúmeras definições para o cálculo fracionário, neste trabalho serão apresentadas as mais utilizadas no campo do controle.

\subsubsection{Definição de Riemann-Liouville}

Inicialmente Liouville definiu uma derivada de ordem arbitrária com uma série infinita, o grande problema dessa definição era que a ordem da derivada deveria ser restrita a valores em que a série convergia. Já Riemann usou uma generalização da série de Taylor a fim de obter uma fórmula para integração de ordem arbitrária. Podem-se unir as duas definições em uma única fórmula. A derivada de Riemann-Liouville (RL) pode ser vista na Eq. 1 [4].

$$
{ }_{a} D_{t}^{\alpha}=D^{n} J^{n-\alpha} f(t)=\frac{1}{\Gamma(n-\alpha)}\left(\frac{d}{d t}\right)^{n} \int_{a}^{t} \frac{f(\tau)}{(t-\tau)^{\alpha-n+1}} d \tau
$$

Onde $n$ é um número inteiro positivo que satisfaz a condição $n-1<\alpha<n, \alpha$ é um número real positivo, $a$ e $t$ são os limites de integração, $\Gamma$ representa a função gama e $J$ é o operador integral, dado pela Eq. 2.

$$
J_{t}^{\alpha}=\frac{1}{\Gamma(\alpha)} \int_{a}^{t} \frac{f(\tau)}{(t-\tau)^{1-\alpha}} d \tau
$$

\subsubsection{Definição de Grunwald-Letnikov}

Grunwald propôs inicialmente um método baseado em uma generalização dos coeficientes de diferenças finitas para derivadas fracionárias. A definição de Grunwald não era matematicamente rigorosa, com isso, A.V. Letnikov demonstrou com riqueza de detalhes e rigor matemático as proposições de Grunwald, obtendo assim a derivada de Grunwald-Letnikov (GL), que pode ser vista na Eq. 3. [5].

$$
{ }_{a} D_{t}^{\alpha}=\lim _{h \rightarrow 0} \frac{1}{h^{\alpha}} \sum_{r=0}^{\left[\frac{t-a}{h}\right]}(-1)^{r} \frac{\Gamma(\alpha+1)}{r ! \Gamma(\alpha-r+1)} f(t-r h)
$$

Onde $n$ é um número inteiro que satisfaz a condição $n-1<\alpha<n$, $a$ e $t$ são os limites do operador.

A integral de GL pode ser vista na Eq. 4.

$$
{ }_{a} D_{t}^{-\alpha}=\lim _{h \rightarrow 0} h^{\alpha} \sum_{r=0}^{\left[\frac{t-a}{h}\right]} \frac{\Gamma(\alpha+r)}{r ! \Gamma(\alpha)} f(t-r h)
$$

\subsubsection{Definição de M.Caputo}

Em 1967, Caputo apresentou em seu artigo uma definição a respeito de derivadas de ordem fracionárias, apresentada na Eq. 5. [6]

$$
{ }_{a} D_{t}^{\alpha}=\frac{1}{\Gamma(n-\alpha)} \int_{a}^{t} \frac{f^{n}(\tau)}{(t-\tau)^{\alpha-n+1}} d \tau
$$

Onde $n$ é um número inteiro que satisfaz a condição $n-1<\alpha<n$, $a$ e $t$ são os limites da integração. 
Abaixo é possível verificar na Tabela 1 um resumo das definições apresentadas e suas principais aplicações.

Tabela 1 - Definições de operadores fracionários e aplicações

\begin{tabular}{|c|c|c|}
\hline & Integral Fracionária & Derivada Fracionária \\
\hline $\begin{array}{l}\text { Riemann- } \\
\text { Liouville }\end{array}$ & $J_{t}^{\alpha}=\frac{1}{\Gamma(\alpha)} \int_{a}^{t} \frac{f(\tau)}{(t-\tau)^{1-\alpha}} d \tau$ & ${ }_{a} D_{t}^{\alpha}=\frac{1}{\Gamma(n-\alpha)}\left(\frac{d}{d t}\right)^{n} \int_{a}^{t} \frac{f(\tau)}{(t-\tau)^{\alpha-n+1}} d \tau$ \\
\hline $\begin{array}{l}\text { Aplicação } \\
\text { (RL) }\end{array}$ & \multicolumn{2}{|c|}{ Indicada para soluções analíticas de funções simples [5]. } \\
\hline $\begin{array}{l}\text { Grunwald } \\
\text {-Letnikov }\end{array}$ & ${ }_{a} D_{t}^{-\alpha}=\lim _{h \rightarrow 0} h^{\alpha} \sum_{r=0}^{\left[\frac{t-a}{h}\right]} \frac{\Gamma(\alpha+r)}{r ! \Gamma(\alpha)} f(t-r h)$ & ${ }_{a} D_{t}^{\alpha}=\lim _{h \rightarrow 0} \frac{1}{h^{\alpha}} \sum_{r=0}^{\left[\frac{t-a}{h}\right]}(-1)^{r} \frac{\Gamma(\alpha+1)}{r ! \Gamma(\alpha-r+1)} f(t-r h)$ \\
\hline $\begin{array}{l}\text { Aplicação } \\
\text { (GL) }\end{array}$ & \multicolumn{2}{|c|}{ Indicada para soluções numéricas de equações diferenciais fracionárias [5] } \\
\hline $\begin{array}{l}\text { M. } \\
\text { Caputo }\end{array}$ & & ${ }_{a} D_{t}^{\alpha}=\frac{1}{\Gamma(n-\alpha)} \int_{a}^{t} \frac{f^{n}(\tau)}{(t-\tau)^{\alpha-n+1}} d \tau$ \\
\hline $\begin{array}{l}\text { Aplicação } \\
\text { (Caputo) }\end{array}$ & \multicolumn{2}{|c|}{$\begin{array}{l}\text { Indicado para aplicações com significados físicos, é a definição mais relevante } \\
\text { para aplicações de engenharia [6], aceita condições iniciais na forma inteira } \\
\left(y(0), y^{\prime}(0), \ldots\right)\end{array}$} \\
\hline
\end{tabular}

\section{Controlador PID fracionário}

O controlador proporcional integral derivativo (PID) é de longe o método mais comum de controlador utilizado em indústrias [7]. De acordo com a pesquisa [8] desenvolvida em 1989 pela Associação de Fabricantes de Instrumentos de Medição Elétrica no Japão (JEMIMA) mais de $90 \%$ dos laços de controle presentes nas indústrias japonesas utilizavam controladores PID, e desses, $80 \%$ apresentavam um desempenho satisfatório. Outra pesquisa realizada em 2001 [9] também mostrou que mais de $90 \%$ dos controladores utilizados em indústrias americanas eram do tipo PID. A razão por trás do domínio dos controladores PID no campo industrial se atribui à sua simplicidade, facilidade de implementação e baixo tempo de estabilização para processos lentos [7].

O Controlador PID fracionário (FOPID - Fractional Order PID) pode ser visto como uma extensão do controlador PID convencional [10]. Os controladores fracionários são menos sensíveis a mudanças de parâmetros de um sistema controlado [11], e por sua vez mais flexíveis no ajuste de ganho e fase, tornando o FOPID uma poderosa ferramenta para o desenvolvimento de sistemas de controle mais robustos, é possível ainda ajustar de maneira mais eficiente propriedades dinâmicas de sistemas de controle [12]. Podemos chamar o controlador PID fracionário de $P I^{\lambda} D^{\mu}$, onde $\lambda$ é a ordem do operador integral e $\mu$ a ordem do operador diferencial. A função de transferência de um controlador $P I^{\lambda} D^{\mu}$ é dada pela Equação (6) [10].

$$
G(s)=\frac{U(s)}{E(s)}=K_{P}+\frac{K_{I}}{s^{\lambda}}+K_{D} s^{\mu} \quad(\lambda, \mu>0)
$$


Onde $G(s)$ é a função de transferência do controlador, $E(s)$ é o sinal do erro, $U(s)$ é o sinal de saída, $K_{P}$ é a constante de ganho proporcional, $K_{I}$ é a constante de ganho integral, $K_{D}$ é a constante de ganho derivativo, $\lambda$ é a ordem do operador integral e $\mu$ a ordem do operador diferencial.

Supondo que $\lambda=1$ e $\mu=1$ obtém-se exatamente a forma de um PID convencional, caso $\lambda=1$ e $\mu=0$ obtém-se um controlador PI, e assim por diante. Com base nessas suposições pode-se inferir que todos os tipos de controladores PID convencionais (PI, PD, PID) são casos particulares de um controlador $P I^{\lambda} D^{\mu}[10]$.

Em comparação a um controlador PID convencional, o controlador FOPID possui dois parâmetros a mais a serem sintonizados, encontrar uma solução ótima para todos os cinco parâmetros em análise $\left(K_{P}, K_{I}, K_{D}, \lambda, \mu\right)$ depende de um processo de otimização de parâmetros em um hiperespaço de cinco dimensões [13]. Com a adição de dois parâmetros, é de se esperar que o controlador FOPID proporcione um desempenho melhor ao sistema de controle. [14].

A sintonia de controladores de ordem fracionária é um dos maiores desafios ao se trabalhar com esse tipo de controlador. Existem inúmeras pesquisas sobre o tema que utilizam uma infinidade de métodos distintos, tais como: Ziegler-Nichols modificado, redes neurais, otimização de enxame de partículas, algoritmos evolutivos, etc. [15-17]

Vários trabalhos ao longo do tempo mostraram áreas e casos onde o controlador FOPID tem um desempenho superior. S. Das et al. [18] e P. Shah [19] mostraram em seus trabalhos que para sistemas de alta ordem o FOPID proporciona melhores resultados que um PID convencional. Vários trabalhos [20-22] demonstraram que para sistemas com um atraso prolongado o FOPID tem um desempenho superior. O PID fracionário também possui maior robustez e estabilidade, como pode ser visto nos casos estudados por [3, 23].

Um dos trabalhos mais importantes utilizando FOPID foi o desenvolvido por I. Podlubny, publicado em 1994. Ele considerou um sistema de ordem fracionária e verificou que um PID de ordem fracionária era mais adequado para o controle do sistema analisado. Foi utilizada a integral absoluta do erro (IAE) como parâmetro de desempenho. Todos os resultados obtidos por Podlubny foram comprovados utilizando métodos numéricos de soluções para sistemas de equações diferenciais de ordem fracionária. O sistema proposto por Podlubny foi simulado para uma resposta ao degrau unitário e ao impulso. [10]. Esse trabalho desenvolvido por Podlubny é considerado como o nascimento do controlador PID de ordem fracionária.

O trabalho de D. Xue et al. [24] publicado em 2006, trás uma análise entre um PID convencional e um FOPID, ambos utilizados para controlar um motor DC, tendo como base dois indicadores de desempenho, a integral do erro quadrático (ISE) e a integral do tempo multiplicado pelo valor absoluto do erro (ITAE), foi possível mostrar a superioridade do controlador de ordem fracionária neste tipo de operação.

Nos trabalhos desenvolvidos no Brasil, pode-se citar o de F.A.C. Ayres Junior et al. [25], utilizando a integral do erro quadrático (ISE) foi possível verificar um desempenho ligeiramente melhor do FOPID em relação ao PID convencional.

Na Tabela 2 encontramos os métodos de controle, as equações e os parâmetros utilizados nos trabalhos acima citados. 
analisados.

Tabela 2 - Resumo dos controladores e equações utilizadas nos trabalhos

\begin{tabular}{c|c|c} 
Nome & Controlador utilizado & Equação do controlador \\
\hline S. Das et al. & $P I^{\lambda} D^{\mu}$ & $0,9007+\frac{1,3198}{S^{0,9495}}+0,3196 S^{0,9284}$ \\
\hline P. Shah & $P I^{\lambda} D^{\mu}$ & $6,3901 e-5+\frac{3,3146}{S^{0,82075}}+6,8983 S^{0,68067}$ \\
\hline V. F. Batlle et al. & $P I^{\lambda}+$ Smith prediction & $\left(-3,71+\frac{1,89}{S^{0,2}}\right)\left(\frac{S+1,52 * 10^{-4}}{S}\right)$ \\
\hline I. Pan et al. & $P I^{\lambda} D^{\mu}$ & $2,5224+\frac{1,4708}{S^{0,9899}}+0,1823 S^{0,7668}$ \\
\hline Y.C. Cheng et al. & $P D^{\mu}$ & $1,0267+0,50 S^{1,09}$ \\
\hline $\begin{array}{c}\text { D. Xue et al. } \\
\text { F.A.C. Ayres Junior et } \\
\text { al. }\end{array}$ & $P I^{\lambda} D^{\mu}$ & $61,57+\frac{91,95}{S^{0,5}}+2,33 S^{0,6}$ \\
\hline
\end{tabular}

\section{Conclusão}

Neste artigo de revisão foi apresentada uma breve introdução sobre a história do cálculo fracionário e suas definições mais utilizadas no campo de sistemas de controle. Foi realizada uma breve análise sobre controladores PID de ordem fracionária, de como representam uma generalização do controlador PID convencional, assim como foram apresentados alguns trabalhos que demonstraram o desempenho superior do FOPID em algumas situações e ainda uma descrição do trabalho de Podlubny, considerado o marco inicial do nascimento do PID de ordem fracionária.

Mesmo sendo comprovada a existência de inúmeros trabalhos a respeito da matéria, o uso de controladores PID fracionários ainda é um tema que necessita de pesquisas extensivas a fim de precisar suas aplicações, capacidades, vantagens e desvantagens nos mais diversos tipos de plantas industriais e aplicações de engenharia.

\section{Referências}

1 D. Cafagna. Fractional calculus: A mathematical toll from the pasto for presente engineers. IEEE Ind Electron Mag. 2007, 2(1), 35-40.

2 M. Axtell; M.E. Bise. Fractional calculus application in control systems. Em: Aerospace and electronics conference, Dayton, 1990, 563-566.

3 C.A. Monje; Y. Chen; B.M. Vinagre; D. Xue; V. Feliu-Batlle. Fractional-order systems and controls: fundamentals and applications. Springer Science \& Business Media, 2010, 414.

4 R. Gorenflo; F. Mainardi. Fractional calculus: Integral and differential equations of fractional order. Em: Continuum Mechanics, Berlin, 1997, 223-276.

5 A. Loverro. Fractional calculus: history, definitions and applications for the engineer. Rapport technique University of Notre Dame: Department of Aerospace and Mechanical Engineering, 2004. 
6 I. Podlubny. Fractional differential equations: an introduction to fractional derivatives, fractional differential equations, to methods of their solution and some of their applications, 198; Academic press, 1998, 340.

7 K.J. Astrom. PID controllers: theory, design and tuning. 2; Instrument Society of America, 1995, 343.

8 S. Yamamoto; I. Hashimoto. Recent status and future needs: the view from Japanese industry. Em: Proceedings of the fourth international conference on chemical process control, Texas, 1991, 1-28.

9 L. Desborough; R. Miller. Increasing customer value of industrial control performance monitoring - Honeywell's experience, em: Proceeding of $6^{\text {th }}$ international conference chemical process control, Tucson, 2001, 172-192.

10 I. Podlubny. Fractional-order systems and fractional-order controllers. Slovak Academy of Sciences Institute of Experimental Physics, 1994.

11 H. Chao; Y. Luo; L. Di; Y.Q. Chen. Roll-channel fractional order controller design for a small fixed-wing unmanned aerial vehicle. Control Engineering Practice, 2010, 18(7), 761-772.

12 Y.Q. Chen; I. Petras; D. Xue. Fractional Order Control- A Tutorial, em: American Control Conference, St. Louis, 2009, 1397-1411.

13 C.A. Monje; B.M. Vinagre; V. Feliu; Y. Chen. Tuning and auto-tuning of fractional order controllers for industry applications. Control Engineering Practice. 2008, 16(7), 798-812.

14 P. Shah; S. Agashe. Review of fractional PID controller. Mechatronics, 2016, $38,29-41$.

15 H. Li; Y. Luo; YQ. Chen. A fractional order proportional and derivative (FOPD) motion controller: tuning rule and experiments. Control System Technology IEEE Transactions. 2010, 18, 516-520.

16 Y. Luo; Y.Q. Chen; Y.G. Pi. Tuning fractional order proportional integral controllers for fractional order systems. Journal Process Control. 2010, 20(7), 823-831.

17 F. Padula; A. Visioli. Tuning rules for optimal PID and fractional-order PID controllers. Journal Process Control. 2011, 21(1), 69-81.

18 S. Das; S. Saha; S. Das; A. Gupta. On the selection of tuning methodology of FOPID controllers for the control of higher order processes. ISA Transactions, 2011, 50(3), 376-388.

19 P. Shah; S. Agashe. Design and optimization of fractional PID controller for higher order control systems, em: International conference of IEEE ICART. 2013, 588-592.

20 V. F. Batlle; R. R. Perez; F. C. Garcia. Fractional order controller robust to time delay variations for water distribution in an irrigation main canal pool. Computers and Electronics in Agriculture. 2009, 69(2), 185-197.

21 I. Pan; S. Das; A. Gupta. Handling pocket dropouts and random delas for unstable delayed processes in NCS by optimal tuning of controllers with evolutionary algorithms. ISA Transactions, 2011, 69(2), 185-197.

22 Y.C. Cheng; C. Hwang. Stabilization of unstable first-order time-delay systems using fractional-order PD controllers. Journal of the Chinese institute of engineers. 2006, 29(2), 241-249.

23 M.S. Tavazoei; M. Haeri. A note on the stability of fractional order systems. Mathematics and Computers in Simulation, 2009, 79(5), 1566-1576. 
24 D. Xue; C. Zhao; Y. Chen. Fractional order PID control of a DC- motor with elastic shaft: a case study, em: Proceedings of American control conference, Minneapolis, 2006, 3182-3187.

25 F.A.C. Ayres Junior; M. C. M. Gomes; E. M. Rocha; W. Barra Junior; J. A. L. Barreiros. Análise experimental de um controlador FOPID aplicado em um regulador de velocidade de um sistema de geração em escala reduzida de 10 kVA. Laboratório de Controle de Sistemas de Potência, Faculdade de Engenharia Elétrica, Universidade Federal do Pará, 2013. 